\title{
B11 - PHARMACOVIGILANCE OF BIOTECHNOLOGICAL THERAPIES: HOW BIO-MANGUINHOS HAS BEEN PREPARING ITSELF FOR THE CHALLENGES THAT HAVE ARISEN?
}

Paulo Roberto Gomes dos Santos¹, Patrícia Mouta Nunes de Oliveira ${ }^{1}$, Hugo Garcia Tonioli Defendi ${ }^{1}$, Maria de Lourdes de Sousa Maia ${ }^{1}$, Maria da Luz Fernandes Leal ${ }^{1}$

1 - The Immunological Technology Institute, Oswaldo Cruz Foundation, Ministry of Health, Rio de Janeiro, Brazil

Objectives: To evaluate the current methods and propose new approaches for optimize the efficacy and safety assessment system, envisioning debates and partnerships for the development of the pharmacovigilance activities on account of the specificities of the biopharmaceuticals.

Methods: Considering the incorporation of drugs to the institute portfolio by "Partnerships for Productive Development (PDPs)" and its commitment with innovation, quality, efficacy and safety, also the Brazilian, European and North American present regulations and initiative projects, a situational analysis of the pharmacovigilance activities have been developed. The current assessment methods for products that have been marketed since 2004 (epoetin alfa and interferon alfa-2b) have been indicated. Confronting these, most adequate methodologies for the products that will be launched on the following years (growth factors, monoclonal antibodies and pegylated molecules) have been sought.

Results: As expected, post-authorisation efficacy and safety studies of paramount importance for public health have been conducted for validating signals from spontaneous reports and active surveillance programs by bibliographic and clinical research. Nevertheless, while Bio-Manguinhos has been leading original and follow-on biologics joint development and technology transfer processes, given the nature of the new products, it has been found that the use of existing practices would not be sufficiently effective for detecting adverse events, and thus minimizing risks. Breaking out of paradigm and invest in new pharmacovigilance approaches is essential before the regulatory requirements and the public opinion about follow-on biologics. The most 
proactive and cost effective strategies should be included on the pharmacovigilance activities. Especially the ones that approach the industries to the opinion former professionals based in health education, research and service institutions. For instance, long-term drug utilisation studies, intensive monitoring schemes, prescription event monitoring, registries and sentinel system that should be placed in a collaborative partnership.

Conclusion: In order to attend the increasing demand of the Ministry of Health, guaranteeing non-charged access of the Brazilian population to effective and safe drugs, including cutting edge biotechnological therapies; and alongside this, overcome the challenges that have arisen, such as the establishment of higher regulatory standards for post-authorisation monitoring in due to the concern regarding the risk-benefit balance of biologics and biosimilars; and theirs acceptance by the organized civil society and scientific community in replacement of known brands that have been sold for more than a decade in the pharmaceutical market, the Pharmacovigilance team at Bio-Manguinhos have been properly preparing themselves for assuring the efficacy and safety of the biopharmaceutical therapies. 